Brief article

\title{
Motor action and emotional memory
}

\author{
Daniel Casasanto ${ }^{\mathrm{a}, *}$, Katinka Dijkstra ${ }^{\mathrm{b}}$ \\ ${ }^{a}$ Max Planck Institute for Psycholinguistics, P.O. Box 310, 6500 AH Nijmegen, The Netherlands \\ ${ }^{\mathrm{b}}$ Erasmus University, Institute of Psychology, P.O. Box 1738, 3000 DR Rotterdam, The Netherlands
}

\section{A R T I C L E I N F O}

\section{Article history:}

Received 3 February 2009

Revised 5 November 2009

Accepted 7 November 2009

\section{Keywords:}

Autobiographical memory

Embodied cognition

Emotion

Language production

Motor action

Metaphor

\begin{abstract}
A B S T R A C T
Can simple motor actions affect how efficiently people retrieve emotional memories, and influence what they choose to remember? In Experiment 1, participants were prompted to retell autobiographical memories with either positive or negative valence, while moving marbles either upward or downward. They retrieved memories faster when the direction of movement was congruent with the valence of the memory (upward for positive, downward for negative memories). Given neutral-valence prompts in Experiment 2, participants retrieved more positive memories when instructed to move marbles up, and more negative memories when instructed to move them down, demonstrating a causal link from motion to emotion. Results suggest that positive and negative life experiences are implicitly associated with schematic representations of upward and downward motion, consistent with theories of metaphorical mental representation. Beyond influencing the efficiency of memory retrieval, the direction of irrelevant, repetitive motor actions can also partly determine the emotional content of the memories people retrieve: moving marbles upward (an ostensibly meaningless action) can cause people to think more positive thoughts.
\end{abstract}

(c) 2009 Elsevier B.V. All rights reserved.

\section{Introduction}

How are the memories we form related to the actions we perform with our bodies? Bodily actions associated with positive and negative emotional valence can influence retrieval of emotional memories. People retrieve memories with positive valence more efficiently when smiling and sitting erect, and memories with negative valence more efficiently when frowning and assuming a slumped body position (Riskind, 1983). Changing participants' body postures can affect how they perform other tasks in the laboratory as well, and how they feel about their performance. In one experiment, participants persisted longer in a puzzle-solving task after assuming an upright posture (Riskind \& Gotay, 1982), and in another they expressed more pride when reflecting on their test scores after sitting upright rather than after slouching down (Stepper \& Strack, 1993).

\footnotetext{
* Corresponding author. Tel.: +310631193013.

E-mail addresses: casasanto@alum.mit.edu, daniel.casasanto@mpi.nl (D. Casasanto)
}

These effects of congruity between action and valence have been interpreted as supporting the hypothesis that emotions are mentally represented, in part, metaphorically. When people talk about emotions they often use expressions that link positive valence with upward motion or position in space (e.g., her spirits soared) and negative valence with downward motion or position (e.g., she's feeling low) (Lakoff \& Johnson, 1999). According to theories of metaphorical representation, these linguistic metaphors correspond to 'mental metaphors' (Casasanto, 2009): nonlinguistic associative mappings from schematic representations in the concrete domains of space and motion (which can be experienced through perceptuo-motor interactions with the physical world), to representations in the relatively abstract domain of emotion (which can only be experienced through interoception or introspection).

Body postures that modulate affective responses in the laboratory tend to have upward or downward trajectories, as do affective facial expressions. Smiling raises the corners of the mouth and eyes, the muscles of the cheeks, and the brow, whereas frowning lowers them. This fact compli- 
cates a metaphorical interpretation of action-valence congruity effects that have been reported previously. According to metaphor theories, smiling and standing tall facilitate positive memories and encourage positive evaluations in part because these bodily actions activate schematic representations of 'upness' that are, by hypothesis, integral to positive emotions. However, it is also possible to explain these action-valence congruity effects non-metaphorically, in terms of basic principles of declarative memory and emotion processing.

According to the encoding specificity principle (Tulving, 1983), recollection is facilitated to the extent that aspects of the retrieval context overlap with aspects of the contexts in which memories were encoded. When a person recalls an event while assuming a similar body position as in the original experience, this congruent body position can improve retrieval of memories related to bodily actions (Dijkstra, Kaschak, \& Zwaan, 2007). Smiling and sitting upright are associated with experiences of feeling happy, proud, or energetic, whereas frowning and slumping are associated with experiences of feeling sad, discouraged, or lethargic. Adopting valence-correlated face or body postures in the laboratory may facilitate positive and negative memories or evaluations by recreating specific aspects of the bodily context in which positive and negative life events were experienced and encoded. Thus, encoding specificity suggests an alternative, non-metaphorical account of previous reports of interactions between bodily action and emotional valence.

The goal of the present study was to determine whether motor actions that are irrelevant to the encoding of emotional memories can still influence their retrieval, consistent with the hypothesized mental metaphor 'positive is up/negative is down'. Rather than adopting face or body postures that correlate with emotional states or convey social meaning (e.g., Tracy \& Matsumoto, 2008), participants performed a meaningless, repetitive motor task while retrieving or recounting autobiographical memories: They moved marbles continually either upward or downward, from one cardboard box to another, timed by a metronome. Actions like smiling and sitting erect habitually co-occur with positive emotional states, but actions like moving marbles repeatedly from one designated location to another do not. Therefore, behavioral effects of congruity between emotional memories and specific emotion-correlated body postures can be readily explained in terms of peripheral feedback or encoding specificity, but effects of congruity between emotional memories and prescribed marble movements cannot. Rather, these congruity effects would provide evidence that mappings from spatio-motor representations to valence are stored in a more schematic format than has been suggested by previous studies, consistent with theories of metaphorical mental representation.

In the first experiment, participants were prompted to retrieve and recount autobiographical memories with either positive or negative valence (e.g. tell me about a time when you felt proud of yourself/ashamed of yourself), while moving marbles either upward (for half of the trials) or downward (for the other half; Fig. 1). We predicted that marble moving would influence autobiographical memory: Participants should retrieve memories faster when the direction of movement was congruent with the valence of the memory they were prompted to tell (i.e., upward for positive memories, downward for negative memories).

In the second experiment participants were given valence-neutral prompts (e.g., tell me about something that happened yesterday), and were instructed to move marbles either upward or downward while retrieving the memory. We predicted that participants would retrieve more positive memories when instructed to move marbles up, and more negative memories when instructed to move them down. Thus, the first experiment tested whether congruity between action and valence influences how efficiently participants can retrieve memories. The second tested whether, beyond influencing efficiency, irrelevant motor actions can also influence the emotional content of the memories people choose to retrieve.

\section{Experiment 1}

\subsection{Participants}

Twenty-four Dutch-speaking undergraduate students participated, in exchange for course credit.

\subsection{Materials}

Participants were seated in front of a laptop computer. On both the right and the left of the laptop were two cardboard boxes, one on top of the other. At the bottom of each box was a tray containing hundreds of clear glass marbles. At the beginning of the experiment, all of the marbles were either in the top trays or the bottom trays, counterbalanced across participants (Fig. 1). One pair of boxes was red and the other blue, and the assignment of colors to the top and bottom boxes was counterbalanced.

A list of 24 memory prompts was constructed, half requiring the participant to recount a memory with positive valence (e.g., Tell me about a time when you felt proud

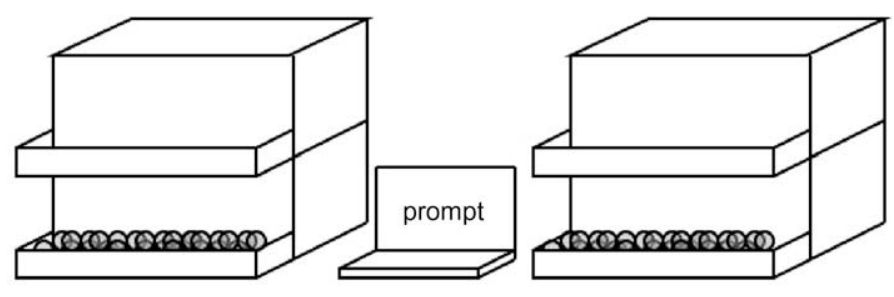

Fig. 1. Schematic drawing of the marble moving apparatus. 
of yourself), and the other half a memory with negative valence (e.g., Tell me about a time when you felt ashamed of yourself). All instructions and prompts were in Dutch, as were the memories participants recounted. English translations of prompts are provided in Appendix A.

\subsection{Procedure}

For each of 24 trials, participants were instructed to move marbles with both hands simultaneously, "into the red box" for one block of trials and "into the blue box" for the other block. Participants moved one marble with each hand every time a metronome sounded, at 2-s intervals. As soon as the metronome started, a prompt appeared on the computer screen instructing the participant to tell either a positive-valence or negative-valence memory. Participants then had $30 \mathrm{~s}$ to retrieve and recount the memory, while moving marbles at the speed of the metronome.

By making the color of the destination box the criterion for marble movement, we drew attention away from the spatial arrangement of the boxes and direction of motion, and avoided using up/down language in the instructions. The box color cued participants to move marbles either upward or downward for the first block of 12 trials, with the direction changing for the second block of 12 trials, ostensibly to avoid running out of marbles. The order of upward and downward blocks was counterbalanced across participants.

Each block contained an equal number of positive and negative memory prompts, pseudo-randomly intermixed. For half of the trials, the direction of movement was congruent with the valence of the memory prompt (upward for positive, downward for negative prompts), and for the other half direction and valence were incongruent.

When debriefed regarding the purpose of the experiment, most participants reported that it was about emotions, or about divided attention. No one guessed that we were testing for a link between emotional valence and direction of motion.

\subsection{Data coding}

Marble movements and memories were recorded with an audio-video camera that was positioned behind the participant. Participants recounted a total of 493 memories, generating a memory on $86 \%$ of the trials. Later, a trained rater evaluated the valence of each memory, blind to the condition in which it had been recounted (congruent vs. incongruent). The valence of the memory matched the valence of the prompt for $98 \%$ of the memories. These trials were analyzed further.

The latency from trial onset to the first word of the participant's memory was determined from the audio-video recording, by a coder who was blind to the valence of the memory that would follow. Trials were removed from further analysis if the response latency was greater than $20 \mathrm{~s}$, leaving less than 10 s to tell the memory ( $0.02 \%$ of the trials).

\subsection{Results and discussion}

Participants began retelling memories faster during schema-congruent movements (i.e., upward movements

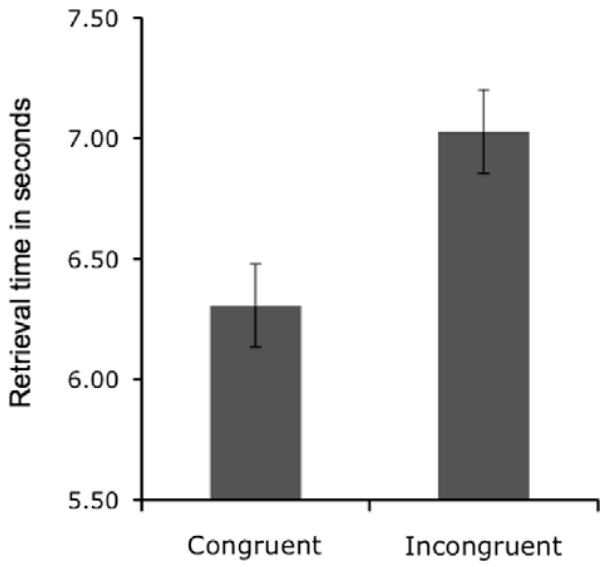

Fig. 2. Results of Experiment 1: latency from trial onset to the start of the memory retelling. Participants began telling memories sooner when movement direction and valence were congruent than when they were incongruent. Error bars indicate s.e.m.

for positive memories and downward for negative memories) than during schema-incongruent movements $(t(23)=2.33, p=.02$, two-tailed; Fig. 2).

The direction of marble movements influenced how efficiently participants produced their memories, consistent with predictions of metaphor theory. Yet, based on this first experiment, it was not clear to what extent the congruity effect arose due to the process of recounting stories aloud as opposed to retrieving them from memory. On a skeptical interpretation, these data might only be informative about the process of planning speech with emotional content, and not about the process of selecting or reconstructing autobiographical memories, per se.

Does motor action influence how people think about emotional experiences, or just how they talk about them (Alibali, Kita, \& Young, 2000; Krauss, 1998)? This question is critical if the results are to be interpreted with respect to mnemonic representations of emotional life events, as opposed to purely linguistic representations of these events. In the second experiment, retrieval and retelling were separated into different phases of the experiment. Participants were instructed to move marbles up or down only during the silent retrieval phase.

The goal of Experiment 2 was to test for a causal influence of motor actions on the valence of autobiographical memories, which could potentially have relevance beyond the laboratory. Can the actions we perform with our bodies partly determine the emotional content of the memories we retrieve? That is, can moving marbles upward (an ostensibly meaningless action) cause people to think more positive thoughts?

\section{Experiment 2}

\subsection{Participants}

Twenty-four Dutch-speaking undergraduates participated, in exchange for course credit. Data were eliminated for two participants. One did not follow the 
instructions. The other had already participated in Experiment 1 (recruited accidentally), and was the only participant in either experiment who, upon debriefing, guessed that the experimental predictions had something to do with a link between the direction of movement and emotional valence.

\subsection{Materials}

The marble apparatus in Experiment 2 was identical to the one used in Experiment 1. Twenty-four new memory prompts were created, all of which were valence-neutral (e.g., Tell me about an event that happened yesterday.) All materials and responses were in Dutch (see Appendix B for English translations of the prompts).

\subsection{Procedure}

As in Experiment 1, participants moved marbles with both hands simultaneously, into the red or blue boxes, every time the metronome sounded, at two-second intervals. However, participants did not retell memories during marble moving. Rather, Experiment 2 was divided into 'retrieval' and 'retelling' phases. During the retrieval phase, participants moved marbles into the designated boxes at the speed of the metronome, while silently reading prompts and retrieving memories. Prompts appeared one at a time in random order for $20 \mathrm{~s}$ each, during which time participants were instructed to think about an appropriate memory. During the retelling phase, participants saw each of the prompts again in the same order, and recounted the memories they had retrieved aloud.

For the first block of trials, participants moved marbles either upward or downward, with the direction changing for the second block. Direction of movement was cued by the color of the boxes, which allowed us to avoid using words like 'up' and 'down' in the instructions. The order of upward and downward blocks was counterbalanced across participants. Retrieval and retelling phases alternated. After each block of 12 retrieval prompts, participants retold the 12 corresponding memories. Participants retold a total of 24 memories, half of which had been retrieved during upward and the other half during downward movements.

\subsection{Data coding}

Marble movements and memories were recorded with a video camera. Participants recounted a total of $489 \mathrm{mem}$ ories, generating a memory on $85 \%$ of the trials. Later, participants rated the valence of each memory: positive, negative, neither (indicating neutral valence), or both (indicating mixed valence). The valence of memories was also evaluated by a trained rater. Inter-rater agreement between participant and coder was $97 \%$. If valence ratings by the participant and the rater did not agree, these trials were removed from further analyses (3\% of trials). Likewise, if the valence of the memory was neither clearly positive nor negative, the trial was removed $(0.02 \%$ of the remaining trials).

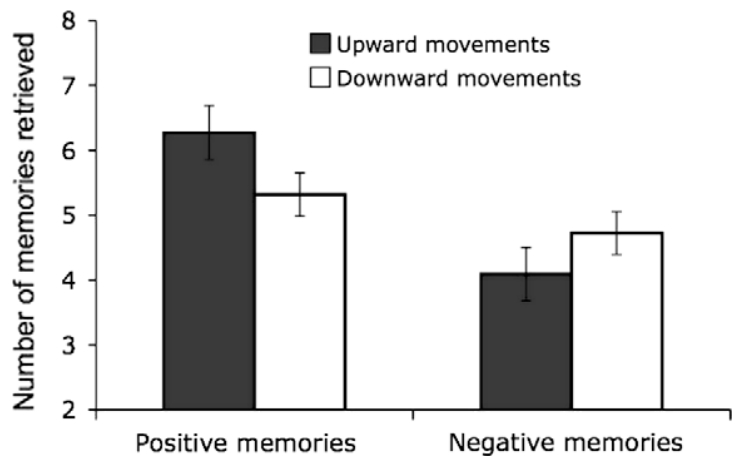

Fig. 3. Results of Experiment 2: effect of movement direction on the valence of memories retrieved. Participants recounted positive memories more often during upward movements (left columns), and negative memories more often during downward movements (right columns). Error bars indicate s.e.m.

\subsection{Results and discussion}

Results of the second experiment showed the predicted interaction between motor action and emotional memory, according to a $2 \times 2$ ANOVA with movement direction (upward, downward) and valence (positive, negative) as within-subject factors $(F(1,21)=5.99, \quad M S e=2.33, \quad p=.02$, partial $\eta^{2}=.22$; Fig. 3). Planned pairwise comparisons showed that, given the neutral-valence prompts, participants produced positive memories more often after making upward movements during retrieval $(t(21)=2.56$, $p=.009$, one-tailed; Fig. 3, left columns), and negative memories after making downward movements $(t(21)=1.60, p=.06$, one-tailed; Fig. 3, right columns). For example, when prompted to recount something that happened in high school, participants were more likely to recollect an experience like winning an award after moving marbles upward, but failing a test after moving them downward.

There was also a marginally significant main effect of valence $(F(1,21)=4.05, \quad M S e=10.45, \quad p=.06$, partial $\eta^{2}=.16$ ), indicating that participants tended to produce more positive memories, overall. The main effect of valence is not relevant to our current hypothesis, but it is consistent with previous reports of positivity biases in autobiographical memory (see Dijkstra \& Kaup, 2005; Mather \& Carstensen, 2005).

\section{General discussion}

Together, these experiments showed that motor actions can influence the retrieval and retelling of autobiographical memories with emotional content. Results support theories of metaphorical mental representation: positive emotions are implicitly associated with upward movements and negative emotions with downward movements. In the first experiment, participants produced memories faster when they moved marbles in a direction congruent with the valence of the memory (i.e., up for positive and down for negative memories). This was true even though 
moving marbles was irrelevant to the task of recounting memories, and vice versa.

In the second experiment, the direction in which participants moved marbles partly determined whether they recounted an autobiographical memory with positive or negative valence. Because participants were randomly assigned to move marbles upward or downward while responding to the neutral-valence prompts, these data go beyond demonstrating a motor-valence correlation; moving marbles upward caused participants to retrieve a greater proportion of positive memories, and moving them downward a greater proportion of negative memories.

These results converge with a growing body of evidence that links bodily action with meaning, memory, and emotion (Barsalou, 1999; Dijkstra et al., 2007; Glenberg \& Kaschak, 2002; Riskind, 1983; Riskind \& Gotay, 1982; Stepper \& Strack, 1993; Tourangeau \& Ellsworth, 1979; Zwaan \& Taylor, 2006). Whereas the majority of studies showing motor-meaning congruity effects have focused on language comprehension, we demonstrate them here in spontaneous language production. Furthermore, whereas Experiment 1 shows that things people do with their bodies can affect how they think (i.e., how efficiently they can produce memories), Experiment 2 shows for the first time that socially meaningless bodily actions can also affect what people choose to think about, at least while they are engaged in conceptual planning for immediate or delayed speech.

But in what sense are emotional memories 'embodied'? According to a version of the embodiment hypothesis that makes testable psychological predictions (Barsalou, 1999), thinking consists in creating mental simulations of bodily experiences, in modality-specific regions of the brain. Knowledge is represented by partial reenactments of sensory, motor, and introspective states, not by amodal redescriptions of these states as suggested by the digital computer-inspired theories of the mind that dominated late 20th century cognitive science (Bower, 1981; Fodor, 1975; Fodor \& Pylyshyn, 1988; Potter, 1979). It is tempting to interpret any data showing links between body and mind as evidence in favor of this hypothesis.

The present data are compatible with embodiment theories, but must be interpreted cautiously with respect to the proposal that emotional memories comprise mental simulations of physical actions. The finding that bodily actions interact with cognition and emotion is not necessarily evidence that cognition and emotion are embodied, in the sense that Barsalou (1999) and others have proposed. Further research is needed to determine whether the spatio-motor components of emotions that we demonstrate here are instantiated by modality-specific mental simulations in perceptuo-motor regions of the brain, as posited by theories of embodied cognition, or alternatively by modality-independent representations as posited by more traditional theories of cognitive architecture.

In both experiments, participants moved their hands from the top box to the bottom box or vice versa on every trial. That is, each action comprised both an upward and a downward movement as participants removed a marble from one box, placed it in the other, and then returned to the original box. Borrowing terminology from gesture research (McNeill, 1992), we can describe the upward and downward marble movements as differing in the directions of their stroke phase (upward during upward marble movements) and retraction phase (downward during upward marble movements). The displacements of the strokes and retractions may have been similar in magnitude, but their goals were different. The finding that stroke directions predicted response times (in Experiment 1) and valence (in Experiment 2) suggests that these congruity effects result from planning or performing goal-directed motor actions.

In order to interpret our results as supporting theories of metaphorical mental representation (i.e., activation of the implicit mental metaphor 'Good is Up'), it is necessary to rule out two potential alternative accounts. First, the theoretical interest of these effects would be diminished if participants were subvocally coding their marble movements as "up" and "down", thus activating positive and negative memories via lexical priming. It is unlikely that this was the case. Participants were never instructed to move marbles upward or downward; rather, they were told to move them 'into the red box' or 'into the blue box'. Therefore, the most task-relevant verbal coding strategy would have been to subvocalize 'red' and 'blue'. But this, too, is highly unlikely, given the requirements of the tasks. Participants' main challenge was to plan a brief verbal description of an episode (in Experiment 2), or to plan and deliver a verbal description (in Experiment 1), under time pressure, while performing a secondary task. Undoubtedly, subvocally coding the directions of marble movements would have interfered with the primary tasks of planning speech and recounting memories out loud making the tasks much harder. Many studies include a verbal interference condition to rule out possible effects of subvocal coding. In our experiments, the primary tasks themselves provided 'verbal interference'.

Second, in addition to the studies reviewed above that link conventionalized up-down motor actions with emotion, a different literature associates flexion movements (generally toward the body) with positive affective and motivational states, and extension movements (generally away from the body) with negative affective-motivational states (e.g., Cacioppo, Priester, \& Berntson, 1993). Importantly, in our experiments upward and downward marble movements required similar combinations of flexion and extension, and similar amounts of motion toward and away from the body. Therefore, it is unlikely that flexion/ extension can explain our results. Emotional valence appears to be linked with the activation of at least three sets of spatio-motor codes: one oriented vertically (as these data show), another related to flexion and extension (as Cacioppo and others have demonstrated), and still another linking positive and negative valence to people's dominant and non-dominant sides of left-right space (Casasanto, 2009). Understanding the interplay of these different codes that associate motion and emotion remains a challenge for future research.

\section{Conclusions}

Motor actions can influence how efficiently people recollect emotional memories, and can also partly deter- 
mine whether people choose to remember episodes with positive or negative emotional valence. Moving marbles upward from one cardboard box to another, a seemingly meaningless action, can cause people to remember more positive life experiences, and moving them downward to remember more negative experiences.

Prescribed marble movements have no social significance, and are not correlated with positive or negative emotions in our everyday experiences. ${ }^{1}$ Thus, unlike previous motor-valence congruity effects, the present results are difficult to explain in terms of encoding specificity (Tulving, 1983) or peripheral feedback from muscles involved in assuming affective facial expressions (Tourangeau \& Ellsworth, 1979) or affect-correlated postures (Riskind, 1983).

Emotional memories not only interact with the specialized body movements that co-occur with positive and negative emotional states (e.g., smiling or frowning, slumping or standing tall), but also with more schematized upward and downward motor actions, as predicted by theories of metaphorical mental representation.

\section{Acknowledgements}

We thank Ilona Boutenstein and Joanne Falconi for help with data collection and coding. Research was supported by NRSA post-doctoral fellowship \#F32MH072502, and by a Grant from the Spanish Ministry of Education and Science \#SEJ2006-04732/PSIC, DGI to D.C.

\section{Appendix A}

Positive- and negative-valence prompts used to elicit memories in Experiment 1. These are English translations of the Dutch stimuli used in the experiment, which will be gladly furnished on request.

Tell me about (positive valence):

... a time you ate something delicious.

... a time when you received a lot of money.

... a time when you won a game or competition.

... a time when someone gave you a compliment.

... a time when you had a lot of fun.

...a time you fell in love with someone.

\footnotetext{
${ }^{1}$ We thank an anonymous reviewer for raising the question whether the action of moving marbles in our task might mimic an action people perform naturally when expressing positive emotions: throwing their hands in the air when they feel victorious (Tracy \& Matsumoto, 2008). Although we cannot definitively rule out the possibility that similarities between marble moving and this spontaneous display of emotion could contribute to the congruity effects we observe, this seems unlikely given the marked differences between these actions. Whereas marble moving involves small, controlled movements within a restricted range, throwing one's arms in the air to express victory usually involves expansive, ballistic movements. Moreover, the 'arms up for victory' gesture is posited to be universally recognizable, but it seems unlikely that an onlooker would construe participants in our marble task to be performing this gesture; when participants were completing an upward marble movement, their hands were raised to about shoulder level, their elbows and wrists were bent, and their fingers were pointing forward making a pincer grip. This posture is not associated with feelings of victory, or with any other conventional expression of emotion.
}

...an important event you experienced

... a time when you received a very nice present.

... an event that made you really proud.

.. a time when you felt really cool.

. an exciting event you experienced.

... a time you accomplished something important.

Tell me about (negative valence):

..a time when something took something valuable from you.

... a time when you felt really sick.

... a time when you had to do something against your will.

... a time when you hurt yourself badly.

... an event during which you reprimanded someone.

. . a time when you were ashamed of yourself.

...a frustrating event that you experienced.

... a time you lost something.

... a sad event that you experienced.

... a time when you were very disappointed in yourself.

. a scary event that you experienced.

... an event that disgusted you.

\section{Appendix B}

Neutral-valence prompts used to elicit memories in Experiment 2. Dutch stimuli furnished on request. Tell me about:

.. a time when you received an unexpected phone call.

... a time when something remarkable happened.

... a time when you received a different grade for a test than you expected.

... a time when someone came to visit you.

... a time when you visited someone.

... a time when you went on a trip.

...something that happened in elementary school.

... something that happened in high school.

...something that happened yesterday.

...something that happened last Summer.

...something that happened before you were school aged.

...something that happened while you were playing sports.

...something that happened while you were on a train.

...something that happened while you were playing outside.

...something that happened while you were talking to someone.

...something that happened in your house.

... an event that involved you and a friend.

... an event that involved you and a family member.

... an event that happened in the evening.

... an event that happened early in the morning.

... an event when you were at your neighbor's.

... an event that happened when it was snowing.

... an event that had to do with water.

... an event that had to do with an animal. 


\section{References}

Alibali, M., Kita, S., \& Young, A. (2000). Gesture and the process of speech production: We think, therefore we gesture. Language and Cognitive Processes, 15, 593-613.

Barsalou, L. W. (1999). Perceptual symbol systems. Behavioral and Brain Sciences, 22, 577-660.

Bower, G. H. (1981). Mood and memory. American Psychologist, 36, $129-148$.

Cacioppo, J. T., Priester, J. R., \& Berntson, G. G. (1993). Rudimentary determinants of attitudes: II. Arm flexion and extension have differential effects on attitudes. Journal of Personality and Social Psychology, 65, 5-17.

Casasanto, D. (2009). Embodiment of abstract concepts: Good and bad in right- and left-handers. Journal of Experimental Psychology: General, 138(3), 351-367.

Dijkstra, K., Kaschak, M. P., \& Zwaan, R. A. (2007). Body posture facilitates retrieval of autobiographical memories. Cognition, 102, 139-149.

Dijkstra, K., \& Kaup, B. (2005). Mechanisms of autobiographical memory retrieval in younger and older adults. Memory and Cognition, 33, 811-820.

Fodor, J. A. (1975). The language of thought. Harvard University Press.

Fodor, J. A., \& Pylyshyn, Z. W. (1988). Connectionism and cognitive architecture: A critical analysis. Cognition, 28, 3-71.

Glenberg, A. M., \& Kaschak, M. P. (2002). Grounding language in action. Psychonomic Bulletin and Review, 9, 558-565.

Krauss, R. M. (1998). Why do we gesture when we speak? Current Directions in Psychological Science, 7, 54-59.

Lakoff, G., \& Johnson, M. (1999). Philosophy in the flesh: The embodied mind and its challenge to Western thought. Chicago: University of Chicago Press.
Mather, M., \& Carstensen, L. L. (2005). Aging and motivated cognition: The positivity effect in attention and memory. Trends in Cognitive Sciences, 9, 496-501.

McNeill, D. (1992). Hand and mind: What gestures reveal about thought. Chicago: University of Chicago Press.

Potter, M. C. (1979). Mundane symbolism: The relations among objects, names, and ideas. In N. Smith \& M. Franklin (Eds.), Symbolic functioning in childhood (pp. 41-65). Hillsdale: L. Erlbaum.

Riskind, J. H. (1983). Nonverbal expressions and the accessibility of life experience memories: A congruence hypothesis. Social Cognition, 2, 62-86.

Riskind, J. H., \& Gotay, C. C. (1982). Physical posture: Could it have regulatory or feedback effects on motivation and emotion? Motivation and Emotion, 6, 273-298.

Stepper, S., \& Strack, F. (1993). Proprioceptive determinants of emotional and nonemotional feelings. Journal of Personality and Social Psychology, 64, 211-220.

Tourangeau, R., \& Ellsworth, P. C. (1979). The role of facial response in the experience of emotion. Journal of Personality and Social Psychology, 37, 1519-1531.

Tracy, J. L., \& Matsumoto, D. (2008). The spontaneous expression of pride and shame: Evidence for biologically innate nonverbal displays. Proceedings of National Academy of Sciences of the United States of America, 105(33), 11655-11660.

Tulving, E. (1983). Elements of episodic memory. New York: Oxford University Press.

Zwaan, R. A., \& Taylor, L. J. (2006). Seeing, acting, understanding: Motor resonance in language comprehension. Journal of Experimental Psychology: General, 135, 1-11. 